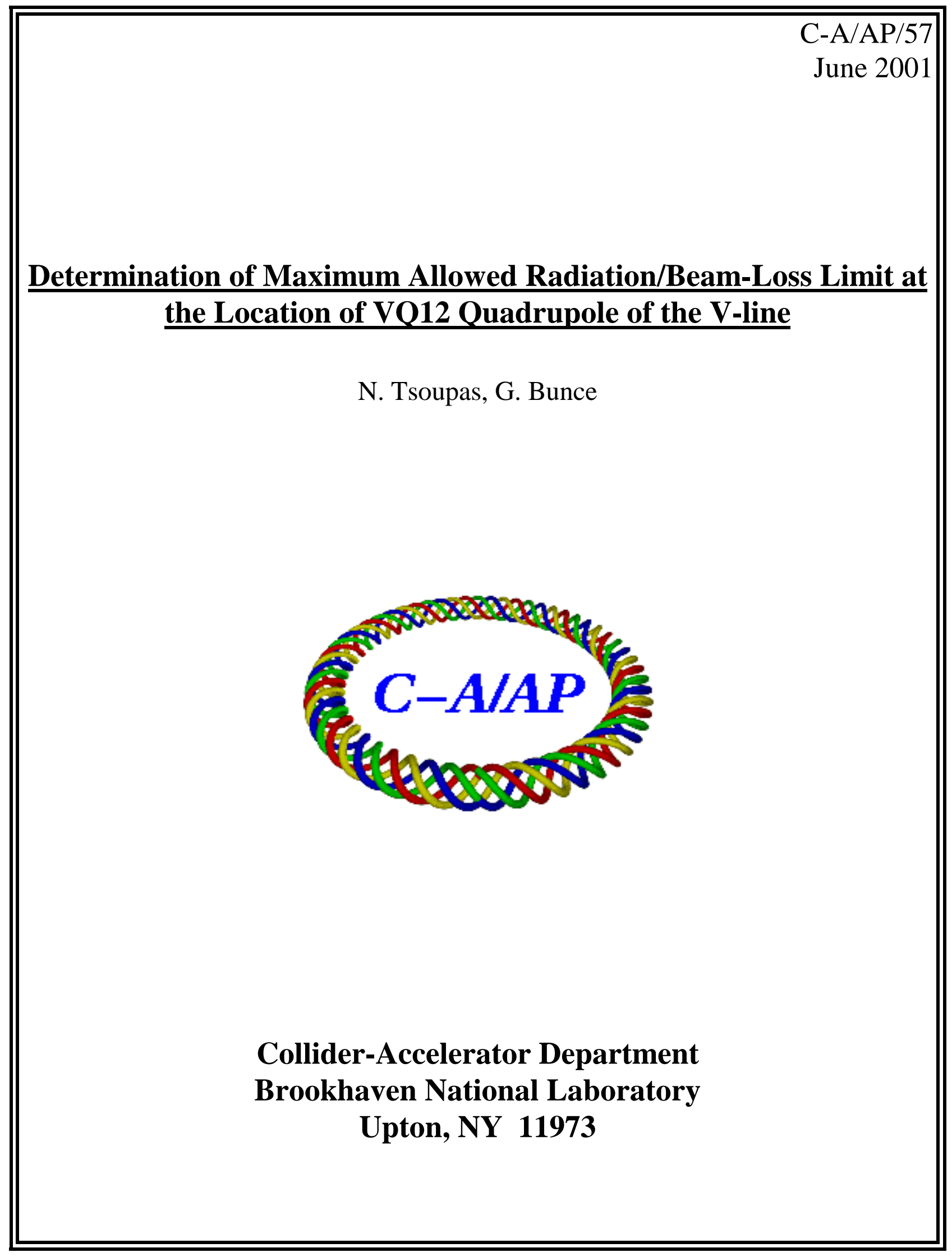




\title{
Determination of Maximum Allowed Radiation/Beam-Loss Limit at the Location of VQ12 Quadrupole of the V-line
}

\author{
N. Tsoupas, G. Bunce
}

\begin{abstract}
The purpose of this technote is to describe the procedure that was followed in order to determine the maximum radiation limit or beam loss limit, to be allowed under the ALARA program, at the radiation ${ }^{2}$ area of the VQ12 quadrupole of the V-line[1]. This radiation limit once exceeded will generate an alarm in the Main Control Room (MCR) which will prompt the MCR members to follow the procedure C-A TPL-99-23 that describes the actions to be taken to reduce the radiation levels below the specified maximum limits.
\end{abstract}

Calibration of VQ12-monitors (as of May $1^{\text {st }} 2001$ ): 0.0014 Tp/count OR 700 counts/Tp

\section{Introduction}

The VQ12 quadrupole of the V-line [1] is a high gradient quadrupole with an aperture of 3". This quadrupole is one of the magnetic elements of the U-V transport line that is utilized in the transport of high intensity proton beam to the V-target [1]. The beam transport optics of the U-V line has been designed by H. N. Brown[1] and a detailed beam TRANSPORT input file appears in Ref [2].

The matrix elements $\left(\mathrm{R}_{11}\right.$ and $\left.\mathrm{R}_{12}\right)$ of the first order transfer matrix (R-matrix) that defines the first order beam transport optics [1,2] from the beginning of the U-V-line to the location of the VQ12 quadrupole, have relatively large values. As a result, small transverse beam variations ${ }^{\amalg}$ (spatial $2 \mathrm{~mm}$ and angular $0.2 \mathrm{mrad}$ ) at the beginning of the $\mathrm{U}-\mathrm{V}$-line, cause large transverse beam variations at the location of the VQ12 quadrupole. These transverse beam variations may cause beam activation of the iron of the VQ12 quadrupole during high intensity proton runs for the $\mathrm{g}-2$ experiment. High radiation levels that have been measured on the quadrupole iron indicate that such activation has happened.

In order to monitor the radiation levels and also to introduce a monitor device to help minimize the amount of beam lost at the VQ12 quadrupole, four radiation monitors [Beam Loss Monitors (BLM's)] were placed at a distance of $8 \mathrm{~cm}$ upstream from upstream face of the VQ12 quadrupole and at a radius $16 \mathrm{~cm}$ from the center of the beam line.

a The radiation at the location of the VQ12 quadrupole is generated when the "halo" of high intensity proton bunches which are transported in the U-V-line to bombard the V-target interacts with the material of the VQ12 quadrupole.

1 Values of the spatial and angular beam displacement at the origin of the U-line, due to the bunch-tobunch variation in the strength of the extraction devices (G10 kicker, H10 septum, AGS extraction Bumps) and beam momentum variations have been calculated and appear in Ref [2]. 
Figure 1 shows a picture of the radiation monitors and the base plate on which the monitors are mounted on. The whole assembly is mounted in front of the VQ12 quadrupole.

The azimuthal location of the four monitors is "top", "right", "bottom" and "left", respectively.

Each of the above BLM's, monitors the radiation that an extracted beam bunch from the AGS may cause when the halo of the beam bunch interacts with the material of the VQ12 quadrupole. The readings of the four BLM monitors provide "relative" information on the radial location of the bunch. This information is used to steer the beam and minimize the beam loss caused by the VQ12 quadrupole.

This technical note describes the calibration method of these four radiation monitors and provides an upper limit which generates an alarm in the MCR. This alarm prompts the MCR personnel to follow a particular procedure to minimize the beam losses below the allowed upper limit.

\section{Calibration procedure of the Four Radiation Monitors at the VQ12 Quadrupole}

\section{Devices used:}

The devices used for the calibration of the of the "VQ12 Radiation Monitors" are shown in figure 2 and are described below:

a) The two VD3,4 dipole magnets which deflect the beam by $3^{0}$ total from the U-line into $\mathrm{V}$-line.

Once the beam is directed into the $\mathrm{V}$-line the beam continues into the $\mathrm{V}$-target with no other bends except the trim magnets.

b) The four "VQ12 Radiation Monitors" which are located in front of the VQ12 quadrupole.

c) The two current monitors, one the uxf1 which is located just downstream from the origin of the U-line, and the other vxf1 which is located just upstream of the Vtarget.

\section{Software used:}

a) A General Purpose Monitor (GPM) which plots the quantities:

$\left\{\sum_{\text {bunch(j) }}\right.$ VQ12(i,j)\}/12 as a function of AGS cycle(time).

Where VQ12(i,j) is the radiation induced by the $j_{\text {th }}$ bunch $(j=1$ to 12$)$ and recorded by the $i_{\text {th }}(i=1,2,3,4)$ VQ12 radiation monitor.

The radiation is caused by 12 beam bunches which are extracted from the AGS and averaged over the 12 extracted beam bunches ${ }^{2}$.

This GPM is shown in figure 3. The radiation monitors are labeled as vlm307.1, vlm307.2, vlm307.3, vlm307.4, and correspond to top, right, bottom, and left respectively.

\footnotetext{
${ }^{2}$ The time interval between extraction of bunches is $\sim 33.3 \mathrm{msec}$.
} 
b) A General Purpose Monitor (GPM) which plots vs. time the following quantities:

b1) The beam current as measured by the uxf1 current transformer located at the beginning of the U-V-line. This current is the average over the 12 beam bunches extracted from the AGS and is labeled as "uxf1" in figure 4.

b2) The quantity $100\{(\mathrm{uxf} 1-\mathrm{vxf} 1) / \mathrm{uxf} 1\}$ (percent difference of current transformers readings).

Where uxf1 is the average beam current ${ }^{3}$ as measured by the current transformer "uxf1" located at the beginning of the U-V-line and vxf1 is the average beam current as measured by the current transformer "vxf1" located at the end of the U-V-line.

b3) The quantity $\Sigma_{\text {mon(i) }}\left\{\left\{\sum_{\text {bunch(j) }}(\operatorname{VQ12}(\mathrm{i}, \mathrm{j}))\right\} / 12\right\} /(\mathrm{uxf} 1)$ :

Where $\left\{\sum_{\text {bunch(j) }}(\right.$ VQ12(i,j)) $\} / 12$ is the quantity mentioned earlier, summed over the four radiation monitors and normalized to the average beam current uxf1.

This GPM is shown in figure 4.

\section{Procedure for the Calibration of the VQ12 Loss Monitors}

a) Optimize the beam transport in the U-V-line to the V-target with a beam current of $\sim 0.75 \mathrm{Tp}$. The goal of the optimization is to produce a reasonable trajectory of the central orbit along the U-V-line with minimum beam losses at the extraction region and the U-V-line itself, by using the available beam-diagnostics (visualflags, BPM's, BLM's) and beam control devices (dipoles and correction dipoles).

b) Allow continuous beam extraction from AGS and beam transport to the V-target; record the quantities mentioned earlier in sub-section 2., and plot them using the GPM's(sub-section 2.).

c) Reduce the strength of the VD3,4 dipole by 60 counts from its nominal value of 2776 counts, and keep on recording and plotting the quantities.

d) Restore the strength of the VD3,4 dipole to its nominal value of 2776 counts, while the beam is transported and the GPM's are plotting the required quantities.

\section{Data Reduction and Results}

The MCR log-book FE 301.1.0001 on pages 50 to 54 contain the details about the Calibration Procedure of the VQ12 loss monitors the Data Reduction and the Results.

Figure 3 shows the GPM which plots, as a function of the AGS cycle, the average radiation $\left\{\sum_{\text {bunch(j) }} \operatorname{VQ12}(\mathrm{i}, \mathrm{j})\right\} / 12$ (j=1 to 12 ) over the 12 bunches for each of the four ( $\mathrm{i}=1$ to 4$)$ radiation monitors.

With the VD3,4 dipole magnet at its nominal setting of 2776 magnet-counts, $\{A=0$ in figure 3 \} the average radiation reading of each VQ12-monitor is $\sim-100$ counts (1mV/count) for a total of $\sim-400 \mathrm{mV}$-counts.

\footnotetext{
${ }^{3}$ The average beam current is measured over 12 extracted beam bunches and is defined in this note as the number of protons in Tp per AGS cycle.

${ }^{4}$ In the rest of this note we will keep the notation of One-VQ12-Loss-monitor count $=1 \mathrm{mV}$-count
} 
These monitor-readings are due to the interaction of the beam halo with the VQ12 quadrupole. When the setting of the VD3,4 dipole magnet changes by -60 magnetcounts, $\{\Delta=-60$ cts on the plot of fig. 3$\}$ the beam is deflected from its optimum trajectory and additional beam halo (even part of the beam) interacts with the VQ12 quadrupole generating more radiation which is detected by the VQ12 monitors.

The gradual increase in the readings of the VQ12-loss monitors (from their minimum value of $\sim-100 \mathrm{mV}$-counts to their maximum value of $\sim-7000 \mathrm{mV}$-counts for a total of all four monitors of $\sim 28000 \mathrm{mV}$-counts), when the magnet setting is reduced by $\Delta=60$ magnet-counts is mostly due to the inductance of the magnet.

Subsequently, at a time 16:00:10 (see x-axis of fig. 2) the setting of the VD3,4 dipole magnet is restored to its nominal value of 2776 magnet-counts, therefore the beam is deflected back to its optimum direction thus reducing the beam losses on the VQ12 quadrupole.

The corresponding reading of the VQ12-monitors are back at the level of $\sim-100 \mathrm{mV}$ counts.

Figure 4 shows the GPM that plots the quantities which were mentioned earlier. These quantities were measure at exactly the same instances of time and under the same conditions, as the quantities shown in figure 3.

The average beam current of the extracted beam which is measured by the current transformer "uxf1" is plotted in figure 4. Note that its value is $\sim 0.75$ Tp per AGS cycle.

The percent difference $100\{(\mathrm{uxf} 1-\mathrm{vxf} 1) / \mathrm{uxf} 1\}$ of the average currents measured by the transformers uxf1 and vxf1 is also plotted in figure 4 . This quantity assumes the value $\mathrm{e}^{\mathrm{b}}$ $-2.0 \%{ }^{4}$, for the setting of VD3,4 magnet corresponding to $\Delta=0$ counts, and the value of $+15 \%$ for the setting corresponding to $\Delta=-60$ magnet-counts (see figure 4 ).

Thus the beam lost on the VQ12 quadrupole when the VD3,4 dipole is set to the value of $\Delta=-60$ is: $17 \% *$ "reading of uxf $1 "=0.17 * 0.75 \mathrm{Tp}=0.13 \mathrm{Tp}$.

From figure 3 the sum of the counts from all the four monitor when when the VD3,4 dipole is set to the value of $\Delta=-60$ is: $28000 \mathrm{mV}$-counts.

Thus the calibration of the VQ12 radiation monitors is:

VQ12_BLM $(\mathrm{cal})=\left(28000\right.$ counts $\left.^{5}\right) /(0.13 \mathrm{Tp})=215(\mathrm{kmV}$-counts $/ \mathrm{Tp})$

Using this calibration for the VQ12_BLM we conclude that the radiation of the $400 \mathrm{mV}$ counts which is detected by all four monitors when the VD3,4 dipole is set to the value of $\Delta=0$ is: $0.002 \mathrm{Tp}=0.2 \%$ of the beam extracted from the AGS.

Details about the calculations above are given in MCR log-book FE 301.1.0001 page 52.

\footnotetext{
5 The negative value of the "percent difference" of the average currents is due to the different calibration constants between the current transformers uxf1 and vxf1. However since we are measuring differences of this "percent difference", the calibration difference is not affecting the calculations.
} 
Recently (g-2 run of year 2001) the readings of the VQ12_BLM's have been converted from $\mathrm{mV}$-counts to "ADC-counts" with the conversion of

(305 mV-counts)/(“ADC-count").

This new conversion modifies the calibration of the VQ12_BLM's from

215 (kmV-counts/Tp) to 700 "ADC-counts"/Tp.

During the g-2 run of the year 2000 we set an upper limit of $12000 \mathrm{mV}$-counts for the sum reading of all four VQ12_BLM's before a warning is issued in the MCR.

In order to keep the same upper limit during the g-2 run of the year 2001 we set the value of 40 "ADC-counts" as the upper limit for the sum reading of all four VQ12_BLM's. This upper limit corresponds to beam loss on VQ12 of $0.056 \mathrm{Tp}$.

A typical plot of the average radiation readings of the four VQ12 monitors as a function of the AGS cycle, is shown in figure 5. During the time these data were collected, the were $\sim 55 \mathrm{Tp}$ of protons extracted from the AGS per magnet cycle. Note the sum of the readings of all radiation monitors $~ 10$ "ADC-counts" or $0.014 \mathrm{Tp}$ (700 “ADC-count/Tp).

\section{Conclusions}

The installation of the four VQ12 radiation monitors has greatly contributed to the ALARA program by providing an additional beam-diagnostics tool to help reduce the beam losses at the VQ12 quadrupole. In addition the reduction of the radiation at the VQ12 quadrupole improves the beam quality on the V-target.

The latest calibration constant (as of May $1^{\text {st }}$ 2001) of each of the VQ12-radiation monitors is:

\section{Calibration: 0.0014 Tp/count OR 700 counts/Tp}

\section{References}

[1] Design Report “A New Precision Measurement of the Muon (g-2) Value” BNL AGS E821 April 1994 page 73

[2] N. Tsoupas et. al. "Fast Extracted Beam (FEB) for the the g-2 Experiment" C-A Tech-note in preparation.

\footnotetext{
${ }^{6}$ The "mV-counts" are converted to "ADC-Counts" and subsequently are multiplied by a factor $\sim 0.001$
} 


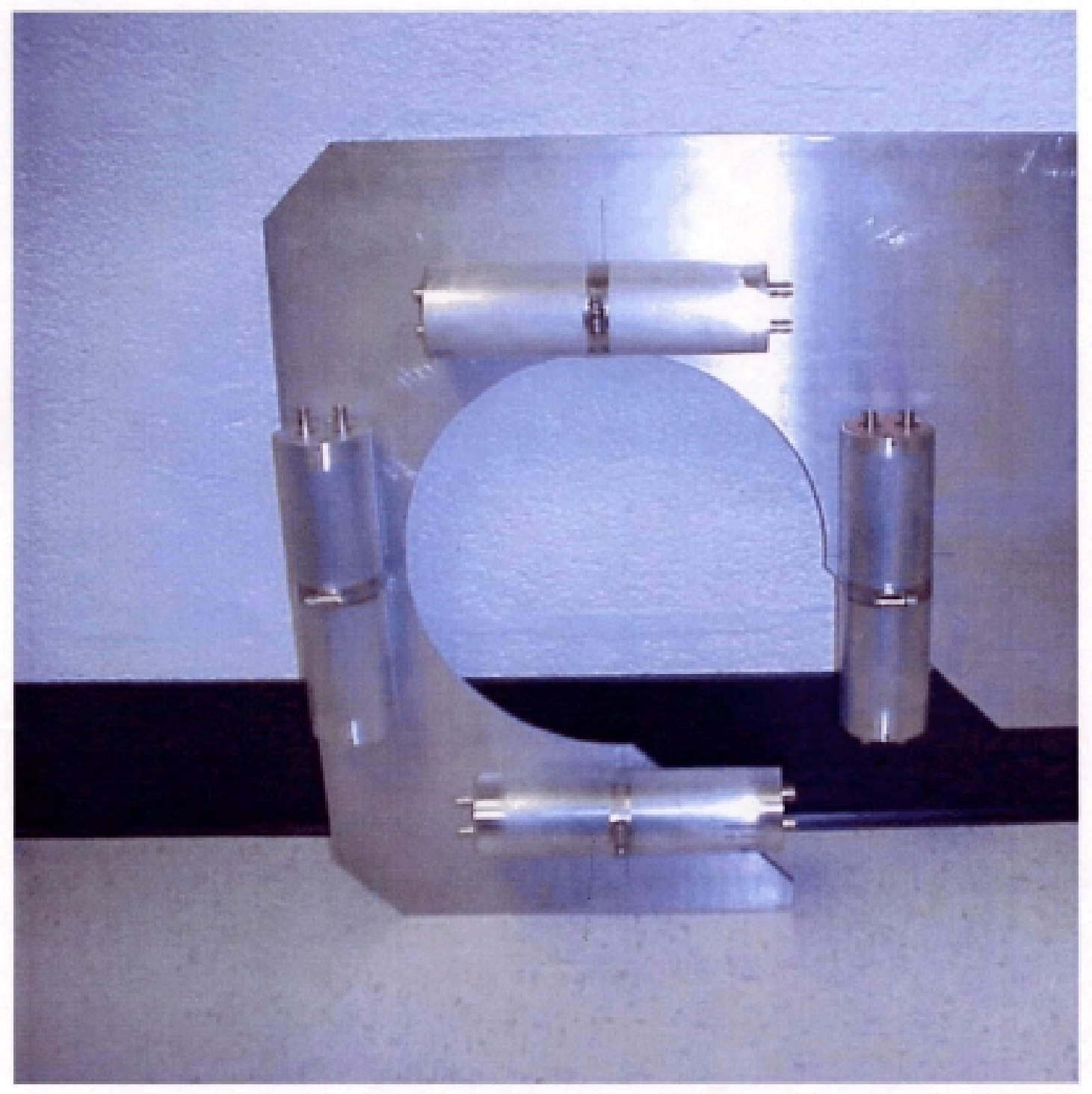

Figure 1: $\quad$ A picture of the four BLM's which are located in front of the VQ12 quadrupole of the U-V-line. 


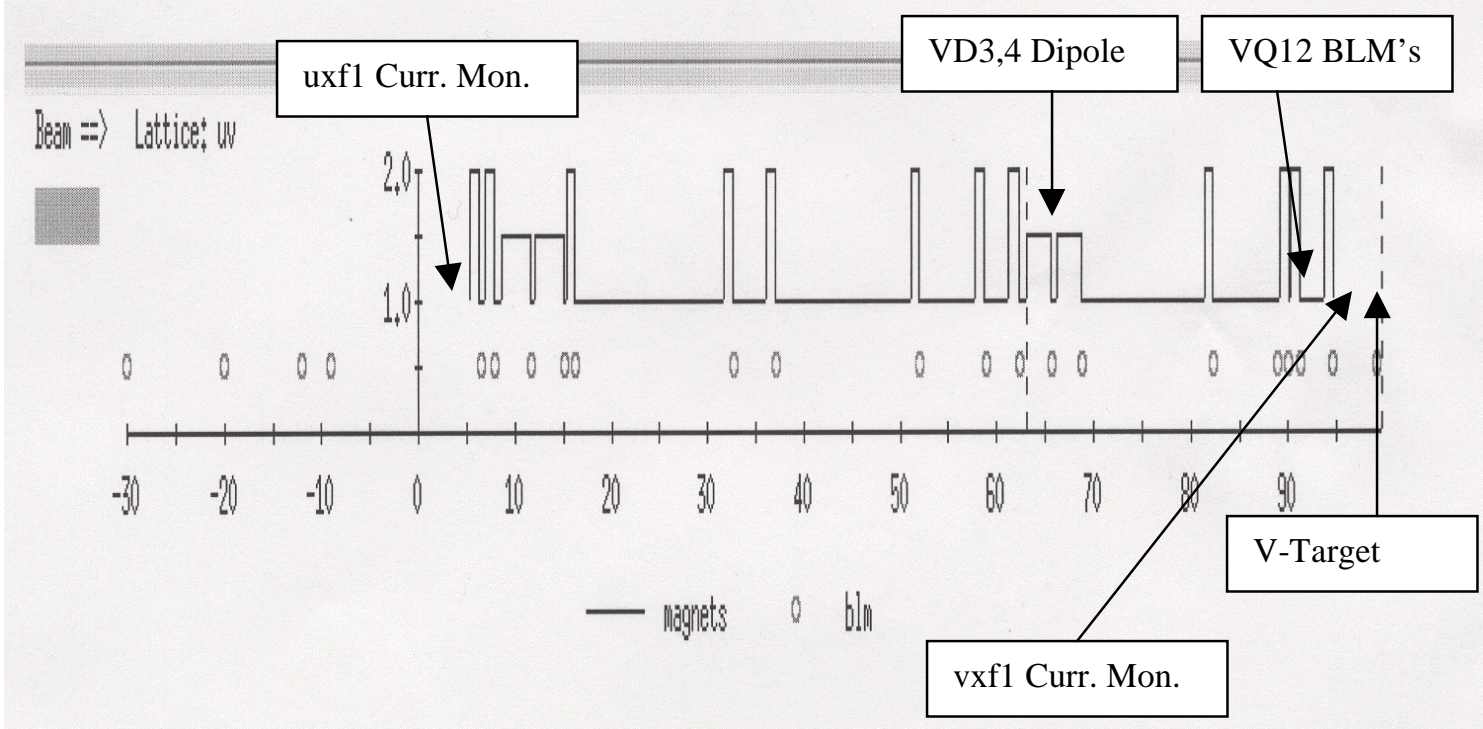

Figure 2. The layout of the magnetic elements of the U-V-line, and the beam diagnostics instrumentation used in the calibration procedure described in the text. The narrow tall boxes correspond to the quadrupoles of the the U-V-line and the shorter boxes to the dipoles. 


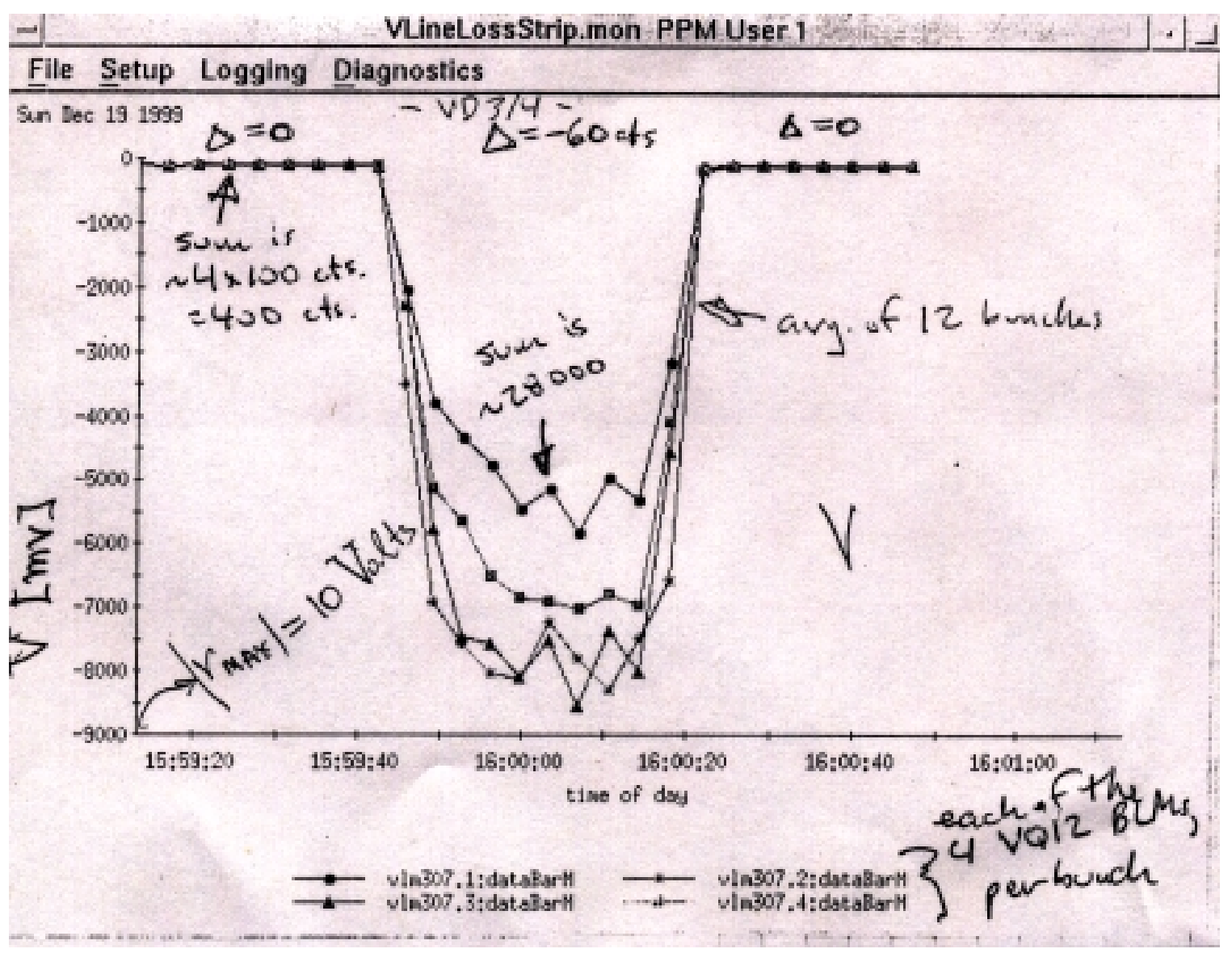

Figure 3. GPM plot of the readings of the four VQ12-BLM's as a function AGS magnet cycle. Note the radiation reading of each VQ12-BLM is $\sim-100 \mathrm{mV}$ counts, for the VD3,4 magnet setting corresponding to $\Delta=0$. The radiation reading has changed to a value of $\sim-7000 \mathrm{mV}$-counts, for the VD3,4 magnet setting corresponding to $\Delta=60$ counts. 


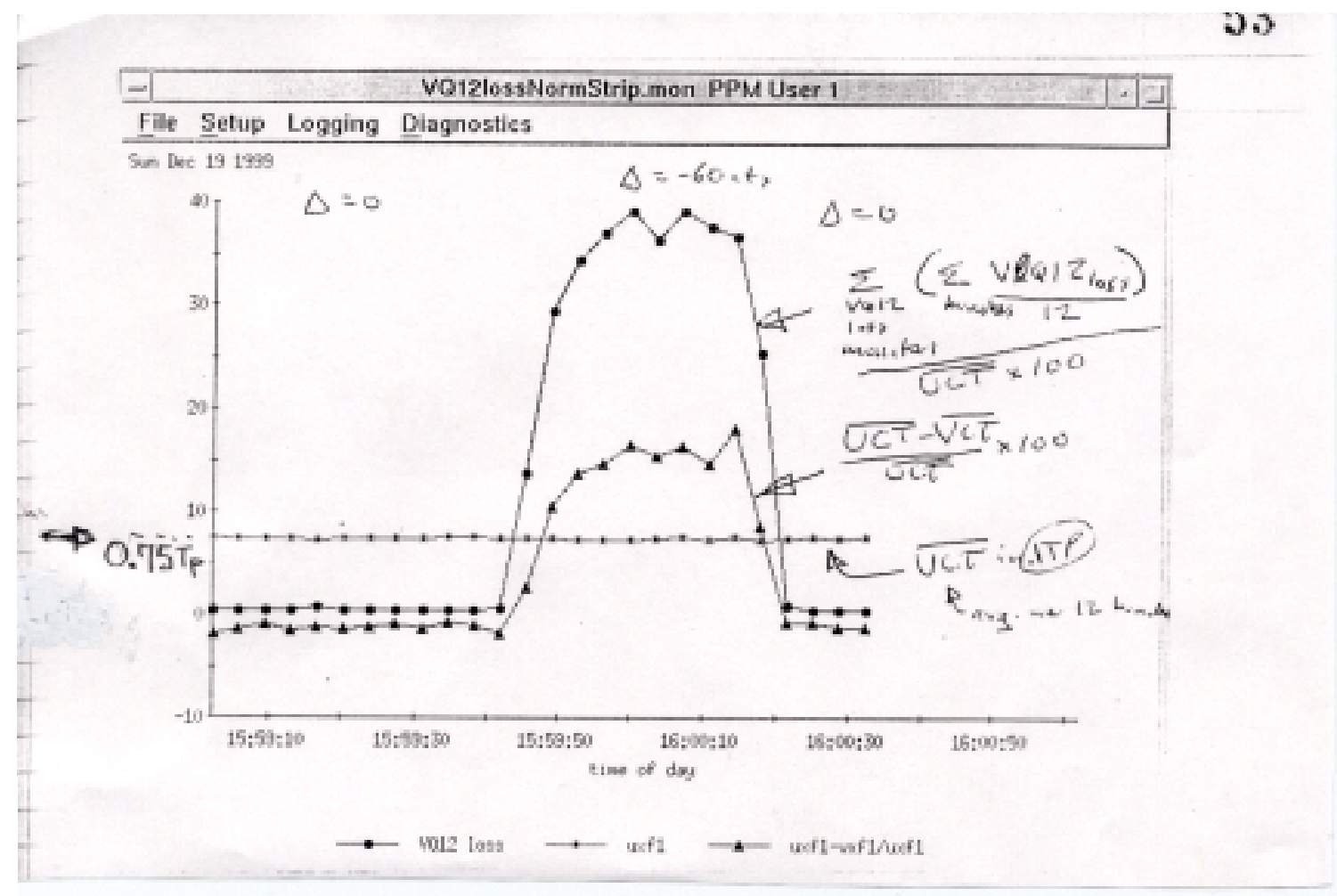

Figure 4. GPM plot as a function of the AGS magnet cycle of the readings:

a) The "uxf1" current transformer

b) The "percent-difference" (see text) between the "uxf1" and "vxf1" current transformers.

c) The sum of the average radiation (see text) of the four VQ12-BLM's normalized to the "uxf1" current. 


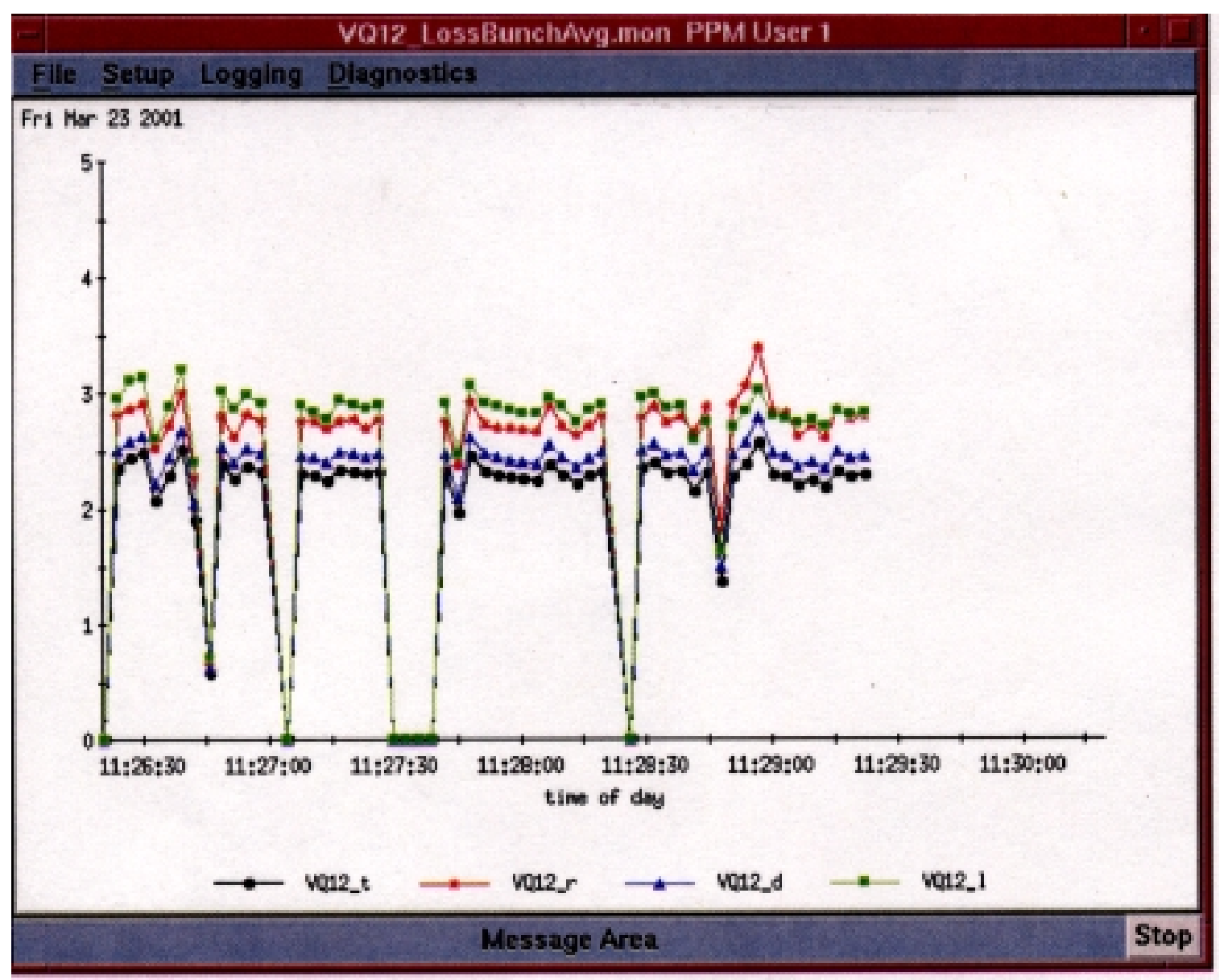

Figure 5. GPM plot of the average reading of each of the four VQ12-BLM's as a function AGS magnet cycle. This is a typical plot of the radiation detected at the VQ12 quadrupole during the g-2 run of the year 2001. The beam extracted from the AGS during the magnet cycle is $\sim 55 \mathrm{Tp}$. The $\mathrm{y}$-scale correspond to 0.0014 Tp/count. 